\title{
ARQUEOMETRIA APLICADA À CONSERVAÇÃO DE SÍTIOS DE ARTE
} RUPESTRE

ARCHAEOMETRY APPLIED TO THE CONSERVATION OF ROCKART SITES

Maria Conceição Soares Meneses Lage

Benedito Batista Farias Filho

Como citar este artigo:

LAGE, Maria da Conceição Soares Meneses; FARIAS FILHO, Benedito Batista. Arqueometria aplicada à conservação de sítios de arte rupestre. In: Cadernos do Lepaarq, v. XV, n.30., p. 327-343, Jul-Dez. 2018. 


\title{
Arqueometria aplicada à conservação de sítios de arte rupestre
}

\author{
Maria Conceição Soares Meneses Lage ${ }^{\mathrm{a}}$ \\ Benedito Batista Farias Filho ${ }^{\mathrm{b}}$
}

\begin{abstract}
Resumo: A Arqueometria tem auxiliado muito a pesquisa arqueológica. No Piauí, tem sido relevante também para auxiliar na conservação de sítios de arte rupestre. Os problemas de conservação existentes nestes sítios são numerosos e variados, de origem natural ou antrópica. É necessário conhecer a constituição química dos pigmentos, do suporte rochoso e de diferentes depósitos de alteração para se realizar diagnósticos e guiar as intervenções. Nesse sentido, tem-se utilizado principalmente aparelhos portáteis de análise ou aqueles que exigem um mínimo de amostras e não as destruam durante os estudos. As principais técnicas de exames e análises utilizados pelo grupo de pesquisa de Arqueometria da UFPI são microscopia portátil, espectroscopia Mössbauer portátil (MIMOS II), Espectrofotometria de Fluorescência X portátil e de bancada utiliza-se Espectrofotômetro Raman e Microscopia Eletrônica de Varredura. Exemplos de estudos são apresentados.
\end{abstract}

\section{Palavras Chave:}

Arqueometria; Conservação; Arte Rupestre.

\begin{abstract}
Archaeometry has greatly aided archaeological research. In Piauí it has also been relevant to assist in the conservation of rock art sites. The conservation problems existing in these sites are numerous and varied, of natural or anthropic origin. It is necessary to know the chemical constitution of the pigments, the rocky support and different alteration deposits to make diagnoses and guide the interventions. In this sense we have mainly used portable analysis devices or those that require a minimum of samples and do not destroy them during the studies. The main techniques of examination and analysis used by the Archaeometry research group of the UFPI are portable microscopy, portable Mossbauer spectroscopy (MIMOS II), portable X-ray Fluorescence and bench-mounted Raman Spectrophotometer and Scanning Electron Microscopy. Examples of studies are presented.
\end{abstract}

\section{Keywords:}

Archaeometry; Conservation; Rock Art.

$\boldsymbol{a}$ Professora Doutora do Curso de Arqueologia e Conservação de Arte Rupestre e dos Programas de Pós-Graduação em Arqueologia e Química da Universidade Federal do Piauí (UFPI), Brasil. E-mail: meneses.lage@gmail.com $\boldsymbol{b}$ Professor Doutor do Departamento de Química e dos Programas de Pós-Graduação em Arqueologia e Química da Univer-sidade Federal do Piauí (UFPI), Brasil. 


\section{INTRODUÇÃO}

O nordeste brasileiro apresenta grande número e diversidade de sítios de arte rupestre, em especial nos Estados do Piauí e da Bahia. Tanto há representações de figurações pintadas, quanto gravadas, com grafismos figurativos e não-figurativos, elaborados com as mais diferentes técnicas e, no caso de sítios pintados, há ampla variedade de cores e tonalidades (Figura 1). No entanto, apenas uma pequena parte deles foi documentada, estudada e publicada, o que é preocupante, considerando o estado avançado de degradação em que alguns painéis rupestres, ou até mesmo sítios, se encontram. Como lembrado por Jacques Brunet (1985), os sítios de arte rupestre são obras de arte expostas ao tempo.
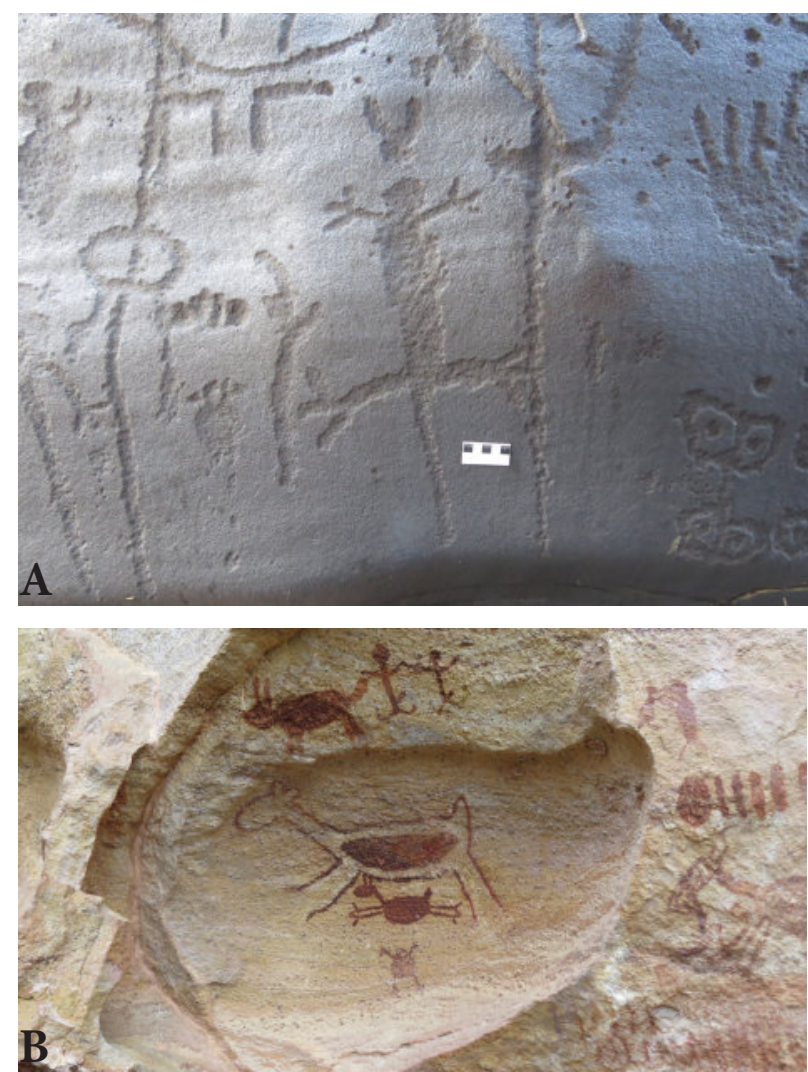
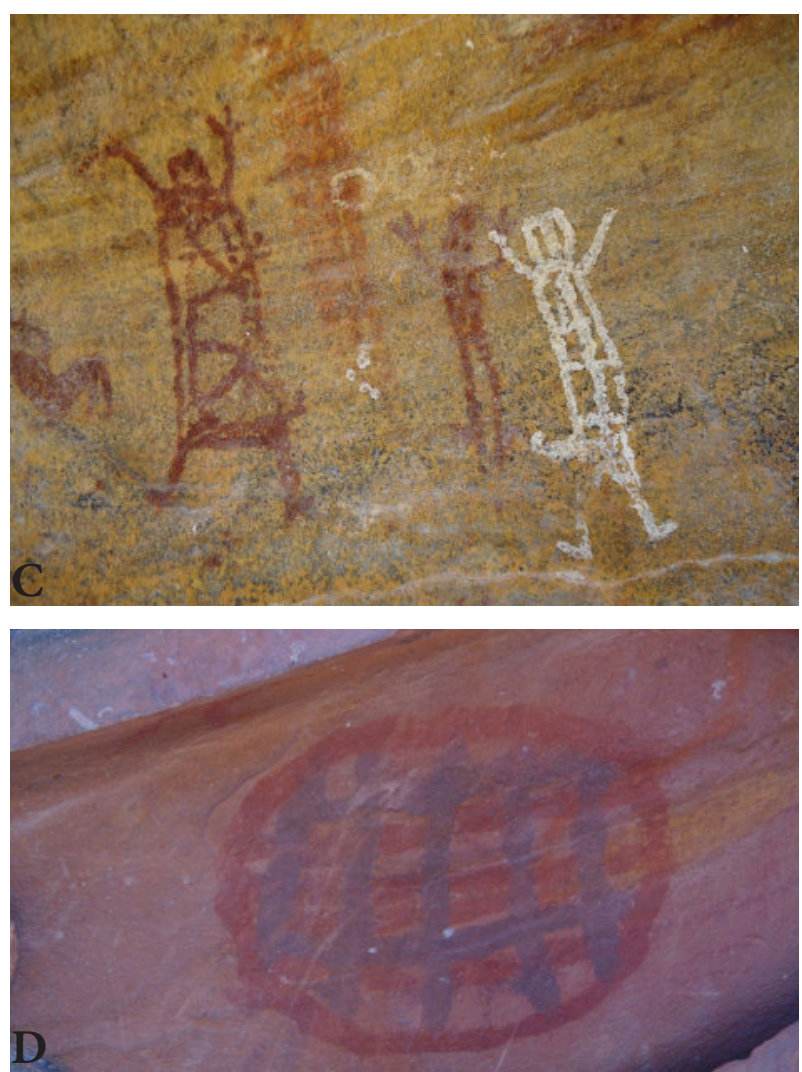

Figura 1: gravuras do Poço da Bebidinha (A); pinturas do PARNA Serra da Capivara (B e C); pintura de Picos dos André-Castelo do Piauí (D) Fotos: Welington Lage (Acervo Pessoal)(A) e NAP ${ }^{1}$ (Acervo NAP) (B; C e D)

Dentre os diferentes problemas de conservação que afetam os sítios, pode-se citar a variação na espessura e profundidade dos sulcos, no caso de gravuras, mudança na cor dos pigmentos, esmaecimento das tintas, migração ou desaparecimento de pigmentos, no caso de pinturas; ou, em ambos presença de eflorescências salinas; fraturas, fissuras, esfoliações do suporte rochoso, fuligens e ataques vândalos.

Ressalta-se que as condições climáticas na área dos sítios são fundamentais para manter o estado de equilíbrio dos mesmos ou favorecer o surgimento de depósitos de substâncias orgânicas ou minerais acumuladas ao longo dos anos, recobrindo a mancha gráfica.

A arqueometria, atuando na interface com as ciências exatas e naturais, utiliza técnicas de exames e análises propondose a responder aspectos direcionados aos mais diversos problemas arqueológicos como função, procedência, tecnologia, 
subsistência, cronologia e outras informações. No que diz respeito à conservação de arte rupestre a arqueometria tem como objetivo investigar e preservar os bens culturais no estudo do homem e de seu meio.

Como forma de diagnóstico, a UNESCO-ICOMOS (ICOMOS, 2016) recomenda a utilização de técnicas analíticas não-destrutivas e não invasivas para as análises arqueométricas, a fim de preservar ao máximo a integridade do material arqueológico. Partindo dessa recomendação, o conservador tem se preocupado em traçar metodologias analíticas de forma que uma pequena quantidade de amostra possa extrair o máximo de informações com as mais diversas técnicas disponíveis, seja de exames ou de análises.

Traçando uma linha do tempo sobre a ciência da conservação, tais estudos eram realizados pelos químicos por meio de métodos clássicos para justificar uma seleção prévia de algum processo de limpeza e conservação. No entanto, devido à necessidade de estudos mais complexos, foram desenvolvidos novos procedimentos analíticos para a caracterização de vestígios da cultura material. Mas sem dúvida o passo decisivo e importante da arqueometria foi o advento de instrumentos portáteis, que permitem desenvolver métodos que dão respostas em tempo real, aumentando a capacidade para tomar decisões ainda no campo (Pérez-Alonso et al., 2004; Maguregui et al., 2011; Roldán et al., 2010).

Além dos vestígios arqueológicos, como os fragmentos cerâmicos, as ossadas humanas, os materiais líticos e as pinturas rupestres, a arqueometria investiga também os depósitos de alteração presentes nos sítios arqueológicos, os quais são verdadeiros "vilões" que ameaçam a riqueza histórica e cultural comuns em sítios de arte rupestre. Tratam--se de depósitos minerais, também chamados de eflorescências salinas, que são agregados cristalinos de sais com composição química variada e formados pela migração de sais solúveis e insolúveis, presentes no interior da rocha, e que são levados pela água de vaporização para a superfície por meio da rede de capilaridade. Esses sais, quando expostos à temperatura do ambiente externo, cristalizam-se, formando uma estrutura de coloração esbranquiçada (Farias Filho et al., 2017; Hennetier et al., 2001; Broggi et al., 2012).

Os problemas de conservação vêm sendo estudados por meio de métodos e técnicas das ciências exatas, ou seja, pela Arqueometria, fornecendo informações sobre os constituintes químicos da rocha suporte, dos pigmentos rupestres e dos depósitos de alteração presentes nos sítios. E a cada dia observa-se a melhoria dessas técnicas, tornando-se menos interventivas e mais precisas.

A arqueometria tem auxiliado as pesquisas arqueológicas complementando os dados dos vestígios e o avanço do conhecimento dos grupos humanos que viveram em uma determinada região, mas também muito vem contribuindo com os estudos sobre o estado geral de conservação dos sítios de arte rupestre, possibilitando a realização de medidas das condições climáticas e ambientais em que se encontram os sítios, identificando a origem dos problemas degradativos e direcionando as intervenções de conservação a serem realizadas.

Análises utilizando técnicas das ciências exatas têm sido realizadas desde os anos 80, pelo grupo de Arqueometria da Universidade Federal do Piauí, cadastrado no CNPq em 1991. Neste mesmo ano, foram iniciadas, de forma sistemática, investigações e intervenções de conservação de arte rupestre em sítios do Parque Nacional Serra da Capivara, sudeste do estado do Piauí.Todavia, no Brasil há outros grupos de pesquisa atuando na área de Arqueometria, dentre eles ressalta-se os trabalhos desenvolvidos e coordenados por Carlos Appoloni, junto ao Laboratório de Física Nuclear Aplicada da Universidade Estadual de Londrina, desde o ano de 1992; as investigações de Dalva Lúcia Faria, da Universidade de São Paulo, e colaboradores, como, por exemplo, Thiago S. Puglieri, da Universidade de Pelotas, que investigam a aplicação da espectroscopia Raman em arte, arqueologia e patrimônio cultural. Há também trabalhos relevantes realizados pelos pesquisadores Helen Jamil Khoury e Henry Lavalle Sullasi, da Universidade Federal de Pernambuco, ligados à metrologia, patrimônio cultural, análise e datação 
de vestígios arqueológicos e Física Nuclear; o professor Luis Antônio Sousa, da Universidade Federal de Minas Gerais, lidera importantes pesquisas de preservação patrimonial, tendo instalado e ampliado o CECOR - Centro de Conservação e Restauração de Bens Culturais; aposentado nesta mesma universidade e Professor Visitante da Universidade Federal dos Vales do Jequitinhonha e Mucuri (UFVJM), José Domingos Fabris e colaboradores desenvolvem importantes investigações em Arqueometria, aplicando a técnica de espectroscopia Mossbaüer na análise e caracterização químico-mineralógica de material arqueológico.

No entanto, a primeira publicação do grupo de pesquisa Arqueometria da UFPI data dos anos 90, é o artigo Premiers resultats de I'étude des peintures rupestres de la Toca do Boqueirão do Sítio da Pedra Furada (Piauí-Brésil) (Brunet e Lage, 1990). No mesmo ano, foi defendida a tese “Étude Archéométrique de l'art rupestre du sud-est du Piauí - Brésil”, de Lage (1990), publicada em 1996 pela Revista de Geologia da UFC, e em 1997, pela Revista do MAE-USP-SP. Todavia a primeira publicação de um trabalho puramente voltado para a conservação de arte rupestre no Brasil data de 1992, é o artigo "Abordagem dos Problemas de Conservação", constante no periódico da UFPI - Cadernos de Pesquisa. Em 2002, Lage, em co-autoria com Niède Guidon, publicaram o artigo "Sauvegarde des peintures préhistoriques d'un site de la Tradition Nordeste” no periódico INORA-França.

Com a consolidação do grupo de pesquisa em Arqueometria, aliado à criação dos cursos de graduação e mestrado em Arqueologia, na UFPI, surgiram muitos trabalhos voltados para a conservação de sítios de arte rupestre. Destaque para os trabalhos de Sousa (2002), que analisou as pinturas rupestres de sítios arqueológicos do Parque Nacional Sete Cidades; Ibiapina (2007), que verificou os depósitos de alteração e as pinturas rupestres do sítio Toca do Joel; Cavalcante (2008), que se dedicou a estudar os depósitos de alteração e pigmentos rupestres de diversos sítios do estado do Piauí; Pinheiro (2009), que analisou as pinturas rupestres e os depósitos de alteração dos sítios Pedra Furada dos Picos e Letreiro do Ninho do Urubu em Castelo do Piauí; T. Alves (2010), que investigou as pinturas de sítios do município de Pedro II; Santos (2010), dedicou-se à análise de pinturas do sítio Salão dos Índios, localizados em Castelo do Piauí; Fontes (2010), que estudou diversos sítios de arte rupestre do Parque Nacional Vale do Catimbau-PE; M. J. Alves (2014), que analisou as pinturas rupestres de sítios do município de São Miguel do Tapuio; Rodrigues (2014), que analisou também as pinturas do Parque Nacional de Sete Cidades; Queiros (2016), que realizou um estudo do estado de conservação dos grafismos rupestres do Médio-Baixo do Jaguaripe-CE e, mais recentemente, Bitú Ferraz (2018), que realizou um diagnóstico técnico de conservação do sítio arqueológico Santa Fé-CE. Muitos desses trabalhos foram apresentados em eventos científicos ou publicados em revistas especializadas (Santos et al., 2018; Cavalcante et al., 2013; Alves et al., 2011; Lage, et al., 2009).

Exemplos da aplicação da arqueometria na conservação do patrimônio arqueológico podem ser encontrados em publicações de periódicos nacionais e internacionais. Dentre esses, tem-se os trabalhos realizados nos parques nacionais Serra da Capivara e de Sete Cidades; no sítio arqueológico Pedra do Castelo, localizado no município de Castelo do Piauí; na Pedra do ingá - PB, no Vale do Catimbau - PE. O presente artigo traz exemplos de trabalhos de conservação realizados pela equipe da UFPI em estudo de diferentes casos, como o sítio Toca do Baixão do Perna II, III e IV, localizados no Parque Nacional Serra da Capivara. 


\section{OS PRINCIPAIS PROBLEMAS DE CONSERVAÇÃO DOS SÍTIOS DE ARTE RUPESTRE}

O primeiro questionamento sobre o estado geral de conservação de um sítio de arte rupestre é tentar descobrir qual era o seu estado original. Fato impossível de ser solucionado, pois o especialista, quando entra em contato com um sítio, já data de centenas ou até mesmo milhares de anos que foi elaborado. Portanto, considera-se como tal o estado em que se encontrava quando foi visitado e documentado pela primeira vez. Neste sentido, pode-se dizer que a grande maioria dos sítios de arte rupestre do Nordeste brasileiro encontra-se em bom estado de conservação, apesar de apresentarem problemas, tanto de origem natural, quanto antrópica. Os problemas de origem natural mais comuns nos sítios do Nordeste brasileiro são ligados à degradação do suporte rochoso, à infestação de insetos construtores, excrementos de roedores que habitam os abrigos rochosos, eflorescências salinas, oriundas da ação das águas nos sítios que as transportam por solubilidade ou arraste e, quando secam, as depositam em superfície ou interstícios da rocha. Já os problemas de origem antrópica, tanto podem ter ocorrido de forma acidental, quanto provocados intencionalmente por reutilizações contínuas do espaço do sítio ou por ações vândalas, sendo estas últimas, na maior parte dos casos, resultantes da falta de informações e conhecimentos a respeito da proteção e conservação do patrimônio arqueológico.

Na Figura 2, são apresentados os diversos problemas de conservação, sejam de origem antrópica sejam natural, encontrados em diferentes sítios de arte rupestre do nordeste brasileiro.

\section{ETAPAS DOS ESTUDOS DE CONSERVAÇÃO DOS SÍTIOS DE ARTE RUPESTRE}

Como apresentados anteriormente, os estudos de conservação de sítios de arte rupestre foram iniciadas, no Brasil, em 1991, no Parque Nacional Serra da Capivara, sob a coordenação de uma das autoras deste estudo, e ampliou tais ações para outros sítios do nordeste. Os trabalhos contaram com a elaboração prévia de um diagnóstico técnico, baseado nos resultados obtidos em exames in situ e análises em laboratórios especializados dos diferentes depósitos de alteração, suporte rochoso e pigmentos rupestres, a fim de entender a origem, a causa e os efeitos da deterioração. Vale a pena ressaltar que nessa etapa, feita preferencialmente em campo, utiliza-se de diversos instrumentos que fornecem resultados em nível microscópico para a caracterização morfológica e da análise químico-mineralógica dos agentes degradantes e da própria arte rupestre.

Logo após a realização do diagnóstico técnico do estado de conservação da arte rupestre, iniciam-se as ações interventivas, que consistem em um trabalho com uma equipe capacitada para retirada ou minimização dos principais agentes de destruição da arte rupestre de um dado sítio. Essa etapa exige muito cuidado, pois se trata do contato direto com os pigmentos rupestres, bens da União e protegidos por lei. São empregadas metodologias já conhecidas e utilizadas pelo Laboratoire de Recherche des Monuments Historiques - LRMH do Ministério da Cultura da França, as quais se utilizam, inicialmente e preferencialmente, de tratamentos físicos como eliminação mecânica e, caso seja necessário, aplicam-se compressas úmidas de água destilada e, de forma mais branda, de soluções químicas para depósitos mais difíceis de serem removidos. Outro trabalho comum nesta etapa refere-se às tentativas de recuperar placas rochosas com arte rupestre, as quais se encontram descoladas da superfície rochosa, aplicando argamassas consolidantes, que também servem para o preenchimento de fissuras ou mesmo recolagem dos materiais desplacados e que ainda se encontram no solo do sítio.

Após a realização dessas etapas, é necessário que o sítio arqueológico seja constantemente monitorado por órgãos preservacionistas, uma vez que se trata de um local a céu aberto e propício a ataques naturais e antrópicos. 

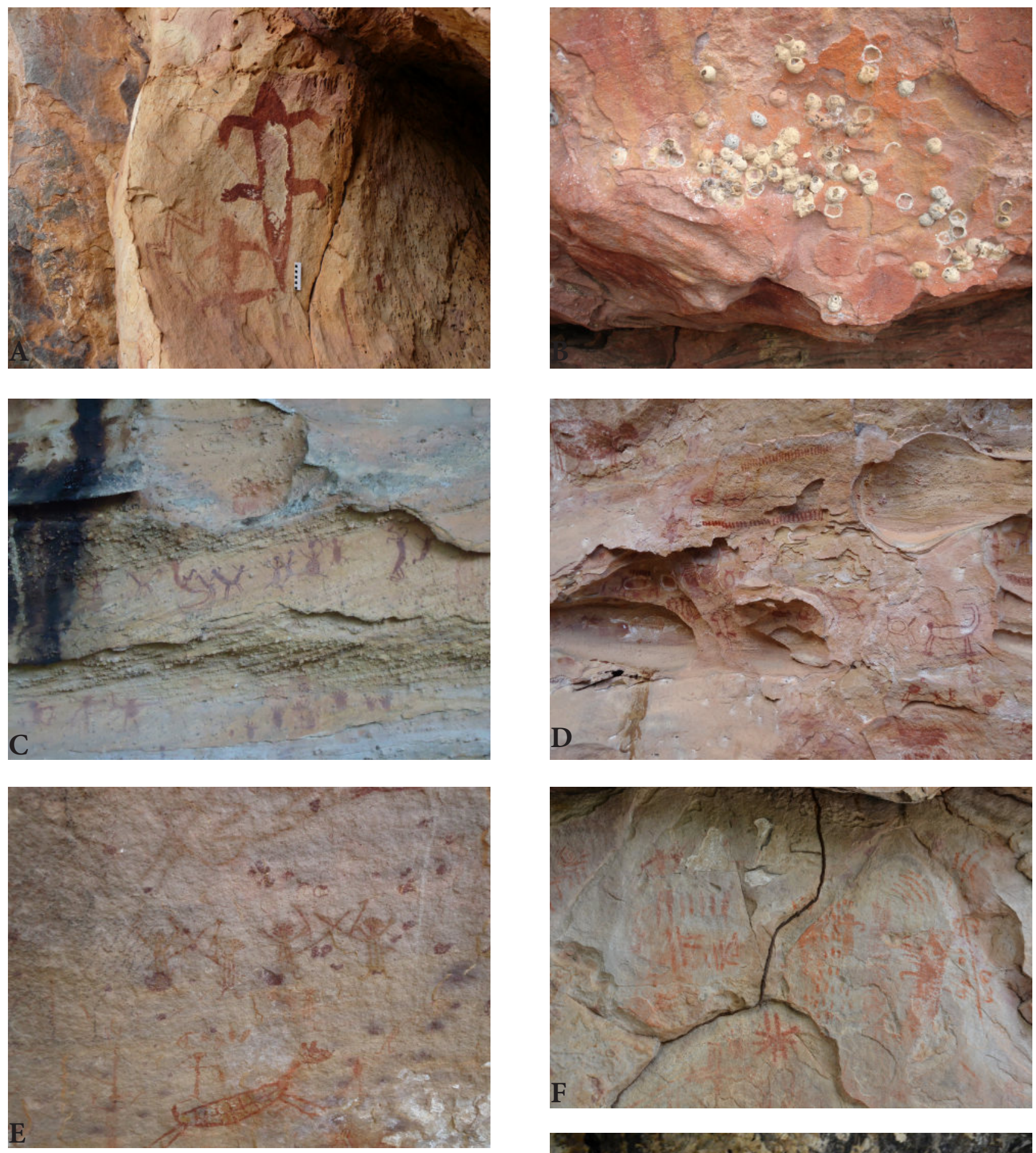

Figura 2: pintura com desgaste antrópico (A); biodepósitos (B; C; D; E e F); desplacamento rochoso (C e D); fuligem sobre pinturas $(G) e$ pichação (H).Fonte:Acervo NAP

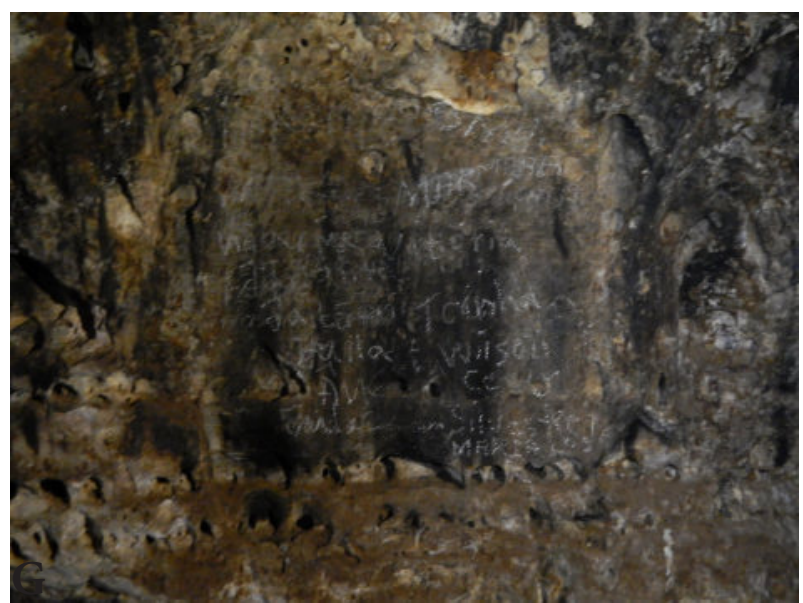




\section{DIAGNÓSTICOS DE CONSERVAÇÃO: ESTUDOS DE CASOS}

A realização de diagnósticos técnicos exige o conhecimento da obra de arte, de sua história e a de seu artista, além do estilo, dos processos de envelhecimento e a deterioração (Brunet, 1985). Portanto, antes de trabalhar com um sítio de arte rupestre é necessário entender a sua origem, os processos e as causas de sua deterioração. Para tanto são feitas análises físicoquímicas tanto dos pigmentos quanto da rocha suporte e dos depósitos de alteração. As técnicas de análises em arqueometria, em conjunto com os exames (microscopia), permitem obter um diagnóstico sobre o estado de conservação da arte rupestre dos sítios em estudo. Até pouco tempo atrás, esses estudos eram realizados empregando técnicas laboratoriais de microanálise, destrutivas, que necessitavam a retirada de amostras dos painéis rupestres. Alguns equipamentos disponíveis (Figura 3) hoje excluem essa parte do trabalho arqueométrico, pois permitem realizar as análises e o diagnóstico em campo, preservando o patrimônio cultural.
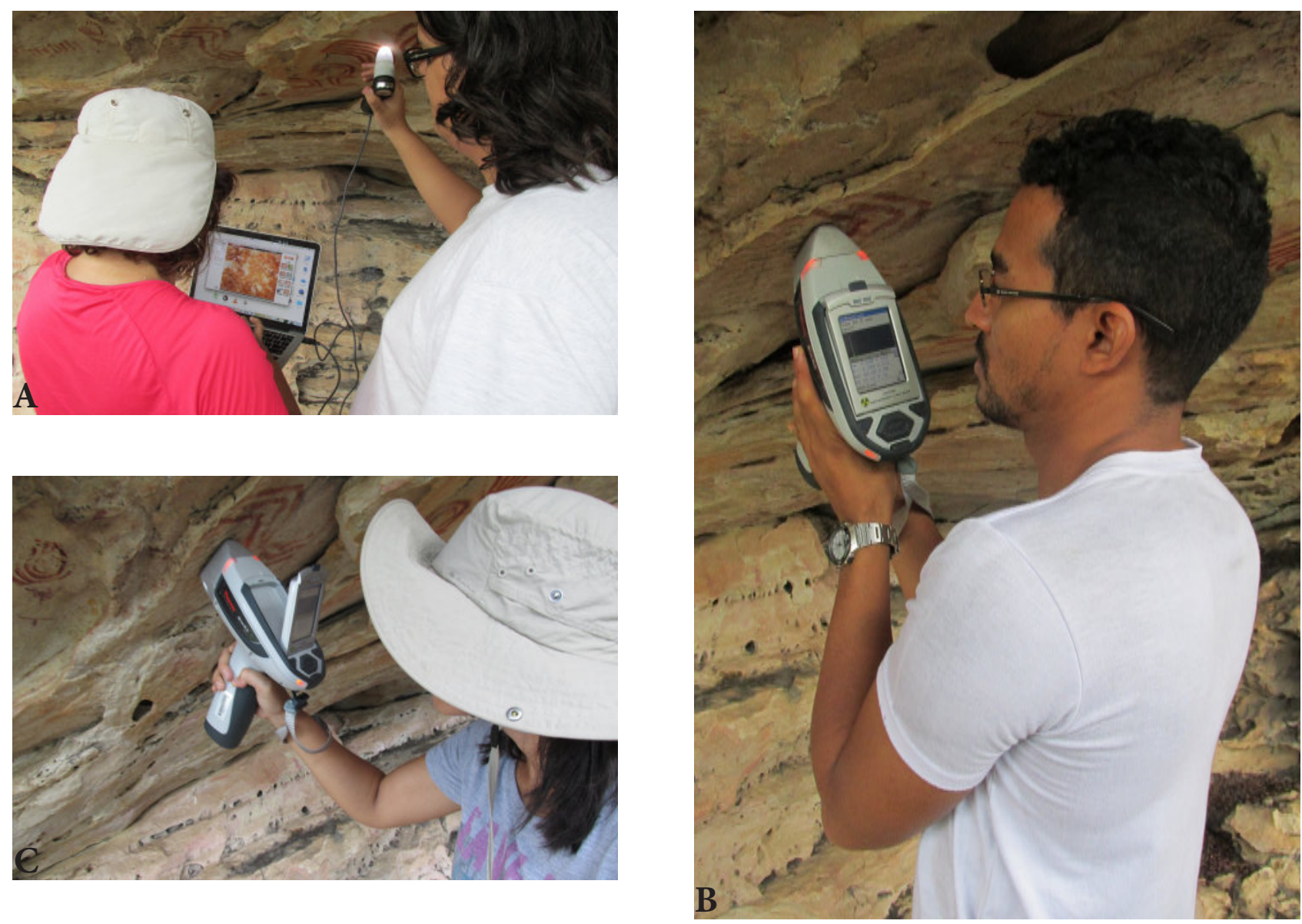

Figura 3: exame com microscopia portátil (A); análise por Fluorescência X portátil (B); medida com código Munsell de cores (C). Foto: Acervo NAP

Apesar de vários esforços que têm sido feitos para contornar ou minimizar o avanço da degradação dos sítios de arte rupestre, sabe-se que é impossível eliminar por completo a degradação destes, e o mais recomendável é que se tomem ações preventivas de conservação, pois são as mais fortuitas e capazes de permitir que esse valioso patrimônio cultural possa chegar a gerações futuras. Para tanto, há um consenso de que, com o avanço das pesquisas arqueométricas, essa preocupação pode ser superada, pois permite, em curto prazo, o estabelecimento de diagnósticos com possíveis estratégias de ações. Desde a década de 90, com os estudos pioneiros na conservação de arte rupestre, os esforços têm se direcionado para caracterizar 
quimicamente os depósitos de alteração de sítios de arte rupestre do Piauí, em especial os da região do Parque Nacional da Serra da Capivara. Como exemplo da aplicação da arqueometria no estudo da conservação de arte rupestre, apresentaremos alguns resultados da análise química dos depósitos de alteração, coletados em diferentes sítios arqueológicos do estado do Piauí, dentre eles as tocas do Baixão do Perna II, III e IV, da Serra da Capivara, e Pedra do Castelo, em Castelo do Piauí (Figura 4). Nos sítios estudados, é possível encontrar muitos depósitos de alteração de origem natural e antrópica. Dentre os naturais, há predominância de eflorescências salinas que, muitas vezes, acabam por recobrir as paredes rochosas ou se encontram muito próximas das pinturas, há ainda problemas de origem antrópica, como fuligem advindas de queimadas muito recorrentes em todo o Piauí.

Quatro amostras de eflorescências salinas foram analisadas: uma do sítio Toca do Baixão do Perna II (BP02.12), duas da Toca do Baixão do Perna III (BP03.05 e BP03.06) e uma da Toca do Baixão do Perna IV (BP04.09). As técnicas de análise utilizadas foram a espectroscopia de emissão óptica em plasma induzida por laser (LIBS) e a espectrometria de absorção no infravermelho com transformada de Fourier (FTIR). As medidas por FTIR-ATR (Agilent Technology, CARY 630 FTIR) foram realizadas prensando uma pequena quantidade das amostras sobre um cristal de diamante. As medidas foram realizadas no intervalo de 4000 a $400 \mathrm{~cm}^{-1}$ com resolução espectral de $4 \mathrm{~cm}^{-1}$, utilizando 64 varreduras. As análises elementares foram realizadas em um equipamento LIBS, constituído por um policromadorechelle (Mechelle 5000, Andor Technology), laser pulsado Nd:YAG $(20 \mathrm{~Hz}, 1064$ nm, FWHM 5,2 ns, e energia máxima do pulso de $110 \mathrm{~mJ}$ ) e detector ICCD (iStar DH734, Andor Technology), empregando energia do laser de $20 \mathrm{~mJ} /$ pulso, tempo de delay de $500 \mathrm{~ns}$ e tempo de integração de $1 \mu$ s.

A Figura 5 mostra os resultados das análises qualitativas obtidas por LIBS, as quais apontam as principais linhas de emissão atômica dos elementos químicos presentes nas amostras.

Apesar dos espectros LIBS apresentarem diversas linhas de emissão atômica, foram selecionadas apenas aquelas mais intensas para realizar a caracterização química da amostra. Como pode ser observada na Figura 5, a linha de emissão de maior intensidade para todas as amostras refere-se ao Ca em 396,8 nm e em $422,70 \mathrm{~nm}$, oriundo do material rochoso no qual se agregam aos depósitos minerais. Pode-se observar ainda outros componentes presentes nas amostras, uns de caráter majoritários e outros em nível de traços. Foram detectadas claramente as linhas de emissão atômica do $\mathrm{Na}$ (dupleto em 588,99 e 589,59 nm), Si $(288,17 \mathrm{~nm}), \mathrm{Al}(309,31 \mathrm{~nm}), \mathrm{Fe}(251,60 \mathrm{~nm}), \mathrm{S}(616,19 \mathrm{~nm}), \mathrm{Mg}(288,17 \mathrm{~nm})$, Ti $(336,11 \mathrm{~nm}$ e 368,52 $\mathrm{nm}), \mathrm{K}(766,49 \mathrm{~nm}), \mathrm{Li}(670,73 \mathrm{~nm})$ e $\mathrm{Cr}(438,38 \mathrm{~nm})$. A triagem desses elementos químicos mostra que, apesar dos mesmos fazerem parte da composição da amostra ou do suporte rochoso, alguns deles podem estar associados a impurezas, uma vez que se trata de amostras retiradas em um sítio arqueológico aberto ao tempo e, por isso, sujeito às ações ambientais, como o vento, a chuva, a poeira, que arrastam materiais até a superfície rochosa.

A fim de se obter a composição química das amostras, foi realizada a análise por FTIR conforme mostra a Figura 6.

Os perfis espectrais por FTIR das amostras revelaram que se trata de uma eflorescência salina bastante similar em composição, apesar de serem sítios arqueológicos distintos. As bandas identificadas no espectro como sendo $1\left(3691 \mathrm{~cm}^{-1}\right)$, $2\left(3618 \mathrm{~cm}^{-1}\right)$ e $3\left(3650 \mathrm{~cm}^{-1}\right)$ referem-se ao estiramento Si-OH. A banda em 4, próxima de $1050 \mathrm{~cm}^{-1}$, refere-se ao modo de vibração de estiramento de Si-O, em $790 \mathrm{~cm}^{-1}$ (6) e em $695 \mathrm{~cm}^{-1}$ (7) a banda de vibração é característica de estiramento Si$\mathrm{Si}$, e em $536 \mathrm{~cm}^{-1}(8)$ corresponde à vibração de deformação de Si-O-Al. O pequeno ombro, em $913 \mathrm{~cm}^{-1}$ (5), é associado ao modo vibracional de deformação do Al-OH. A banda larga em aproximadamente $3400 \mathrm{~cm}^{-1}$ é devido ao modo de estiramento de hidroxila da água. Essas informações permitem identificar que as amostras dos sítios em estudo são compostas de caolinita $\left[\mathrm{Al}_{2} \mathrm{Si}_{2} \mathrm{O}_{5}(\mathrm{OH})_{4}\right]$ (Alencar et al., 2015; Saika et al., 2008). 

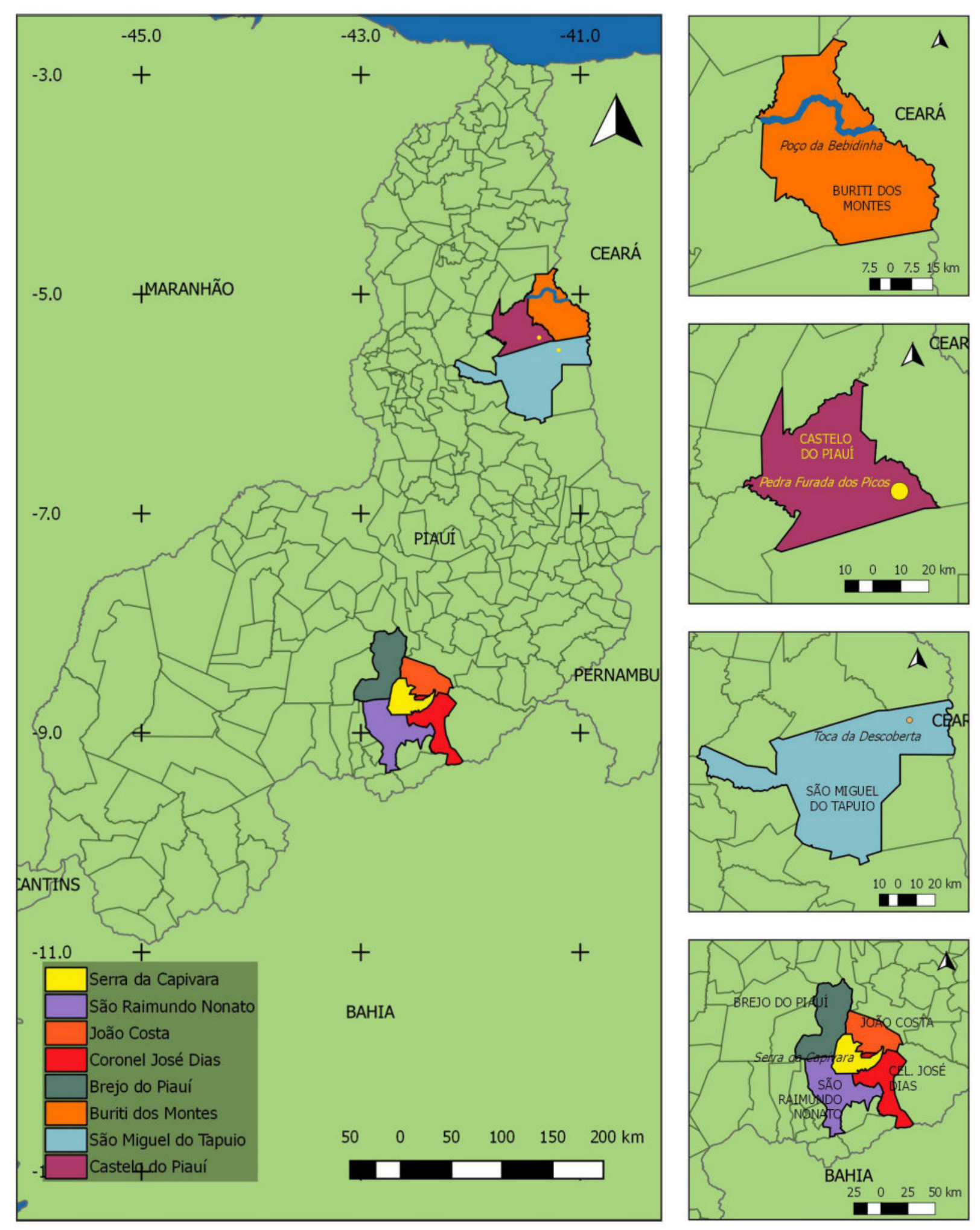

Figura 4: Mapa de localização dos sítios. Foto: Wellington Lage (Acervo Pessoal) 

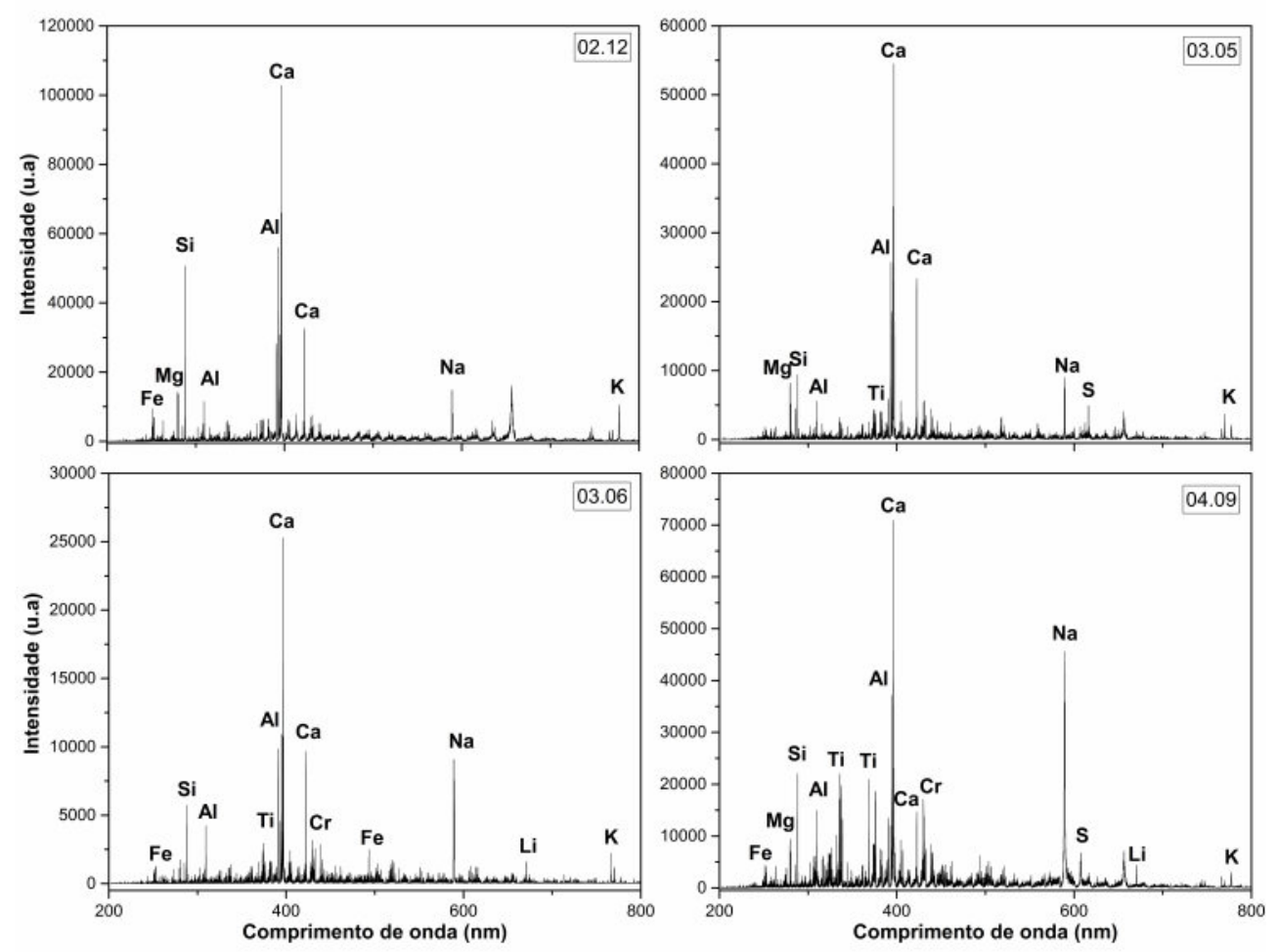

Figura 5: Linhas de emissão atômicas obtidas por análises LIBS das amostras de eflorescência salina dos sítios Toca do Baixão do Perna II, III e IV.

Os resultados obtidos para as amostras de eflorescências salinas dos três sítios arqueológicos direcionam para os trabalhos de conservação a serem realizados nos sítios em estudo, uma vez que, conhecendo a natureza química dos materiais, torna-se mais viável a aplicação de estratégias físicas e químicas para remoção ou minimização desses materiais nos paredões de arte rupestre.

Outras técnicas analíticas também têm sido utilizadas pelo grupo de Arqueometria da UFPI, a fim de efetuar diagnósticos técnicos de conservação de sítios de arte rupestre, como, por exemplo, a espectroscopia de fluorescência de raios X portátil e a espectroscopia Mossbaüer portátil (MIMOS II). Estas bem mais empregadas para identificação da composição químico-elementar e mineralógica de pigmentos pré-históricos, todavia de essencial importância para a realização de futuras intervenções de conservação. Como exemplo, apresentamos o caso de análises fluorescência de raios X portátil no sítio Pedra do Castelo em Castelo - Piauí.

A Fluorescência de Raios X é uma técnica analítica qualitativa e semi-quantitativa, que usa a interação dos raios X com a amostra a fim de se obter a sua composição química elementar. Esta técnica apresenta potencialidades para a análise de material arqueológico por não necessitar de preparo de amostra, é não-destrutiva e não invasiva e sendo possível realizar medidas in situ, sem a necessidade de coleta de amostras (VANDENABEELE e DONAIS, 2016). Foram analisadas amostras de depósitos de alteração (fuligem) que se encontravam no paredão rochoso, bem como de um pigmento de cor vermelho, do sítio arqueológico Pedra do Castelo. As amostras foram analisadas diretamente sob o suporte rochoso, utilizando um espectrômetro de FRX (Thermo Fisher Scientific, Niton XL3t Ultra), equipado com tubo de raios X com um anodo de prata e um detector de deriva de silício (SDD). O equipamento possui um tubo de raios $\mathrm{X}$ de voltagem máxima de $50 \mathrm{kV}$, corrente de $200 \mu \mathrm{A}$ e $2 \mathrm{~W}$ de potência e uma câmera CCD acoplada, que permite visualizar o ponto no qual foi feita a medida e registrá-lo junto ao resultado analítico. As amostras foram analisadas utilizando um ponto focal de $3 \mathrm{~mm}$ de diâmetro e cujo tempo total de análise foi de 120 segundos, realizada sob atmosfera. 


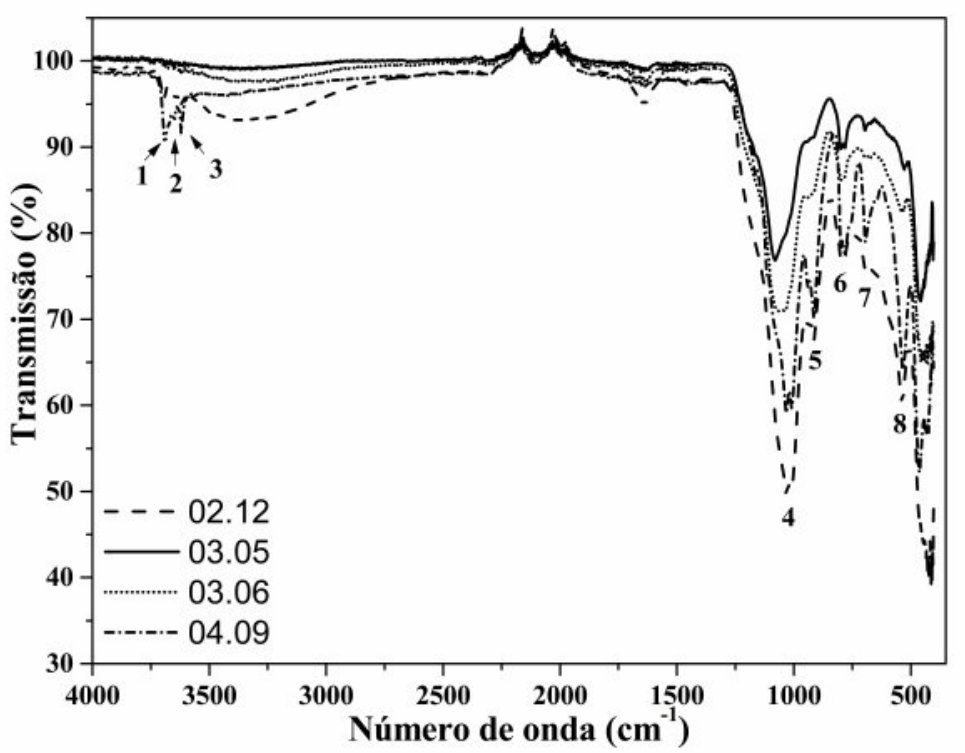

Figura 6: Linhas de absorção por FTIR das amostras de eflorescência salina dos sítios Toca do Baixão do Perna II, III e IV.

A Figura 7 apresenta os resultados das análises de Fluorescência de Raios X portátil do depósito de alteração da rocha e de um pigmento de cor avermelhada, presentes nas paredes rochosas do sítio em estudo.

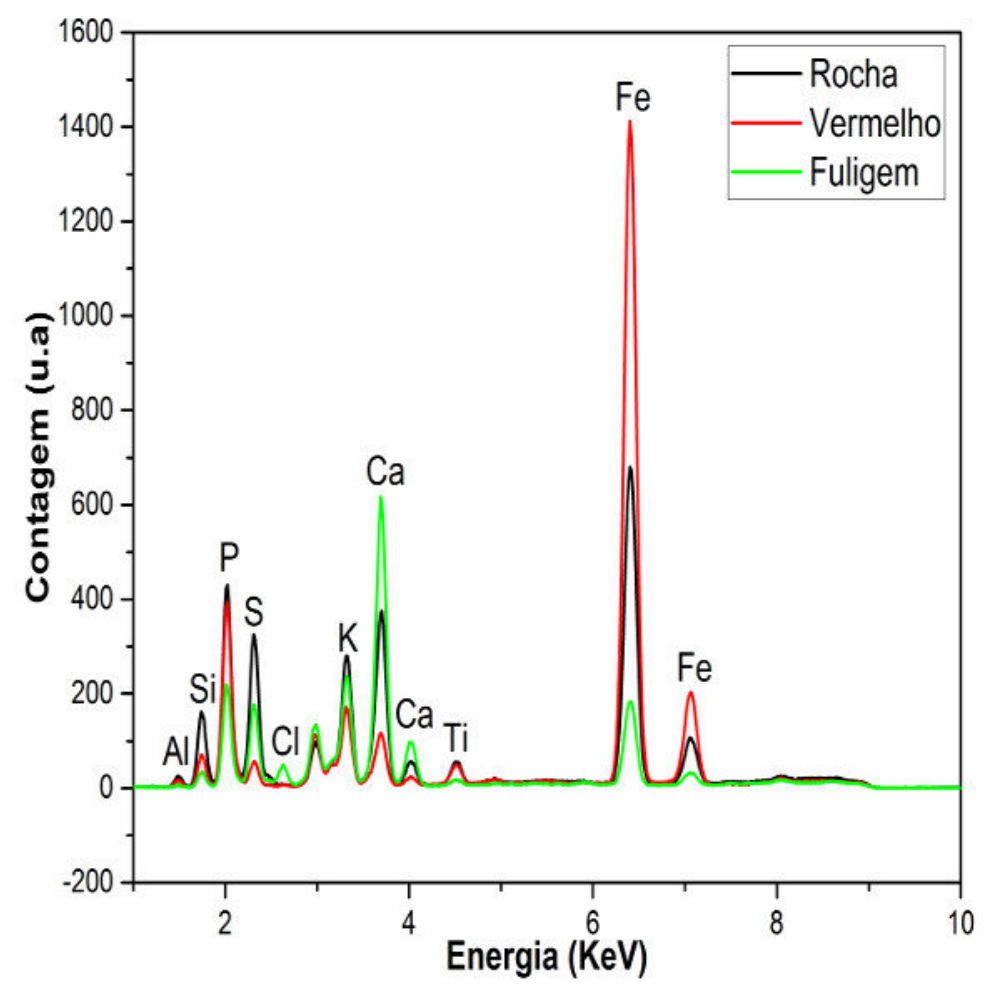

Figura 7: Espectro de Fluorescência X portátil

Como se pode observar, há uma composição bastante similar entre rocha, pigmento e depósito de alteração, sendo apenas cloro como elemento diferenciador, pois se encontra apenas na composição da fuligem. Esta forneceu ainda importante concentração de cálcio, potássio, enxofre e fósforo. A presença do fósforo no pigmento vermelho pode indicar uma possível contaminação com tal biodepósito presente na área analisada. 
Além desses estudos, ressalta-se ainda outros trabalhos também realizados por nosso Grupo de Arqueometria da UFPI, utilizando Espectroscopia Mössbauer portátil (MIMOS II), como a análise dos pigmentos vermelho claro e vermelho escuro do preenchimento do corpo do cervídeo maior, figura símbolo do Parque Nacional Serra da Capivara (Figura 1b), e o resultado forneceu duas composições distintas de óxido de ferro, sendo a mais escura uma mistura de duas hematitas, uma delas com composição bem mais cristalizada (Lage et al. , 2016). Outros trabalhos têm sido focados na caracterização químico-mineralógica de pigmentos e utiliza a espectrometria Mössbauer como complementar às outras técnicas analíticas, como os trabalhos realizados no sítio Salão dos Índios, em Castelo do Piauí (Santos et al., 2018), e do sítio Manantial Solís, localizado na Patagônia, Argentina (Cavalcante et al., 2017).

É importante lembrar também que, além das técnicas analíticas, há ainda as técnicas de exames, que constam de observações microscópicas em campo, sob a ocular de lupas de diferentes aumentos (10X; 30X; 50X; 100X.). Em estudos anteriores, tais exames possibilitaram a elucidação de alguns casos tidos como intrigantes para a pesquisa arqueológica, como, por exemplo, a presença do elemento ferro na composição química do pigmento cinza, de sítios da Serra da Capivara (Lage et al. 2004). Esses exames permitiram constatar que tal pigmento é constituído de uma mistura de caolinita (branco) e hematita (vermelho). Outro fato elucidado aconteceu com o pigmento azul, do sítio Toca dos Veadinhos Azuis, também da Serra da Capivara. A observação microscópica mostrou que esta cor azulada foi obtida em consequência da deposição de uma camada de sal à base de silício, de cor esbranquiçada e translúcida, sobre uma camada de pigmento preto, constituído de carvão vegetal (Lage, 2007).

Além disso, a microscopia óptica portátil também tem fornecido importantes resultados sobre os depósitos de alteração presentes nos sítios, contribuindo para a elaboração de diagnósticos técnicos sobre o estado geral de conservação de sítios de arte rupestre, como, por exemplo, os que foram realizados no sítio Pedra do Castelo em Castelo - Piauí, em 2017, e pode-se observar a presença de pigmentos vermelhos embaixo de uma camada de fuligem (Figura 8A), indicando o recobrimento de antigas pinturas rupestres por esse depósito, e, em outro, a presença de recentes pichações em argila vermelha, recobrindo uma antiga, efetuada com tinta a óleo amarela, que recobria uma gravura rupestre pintada em preto (Figura 8B).
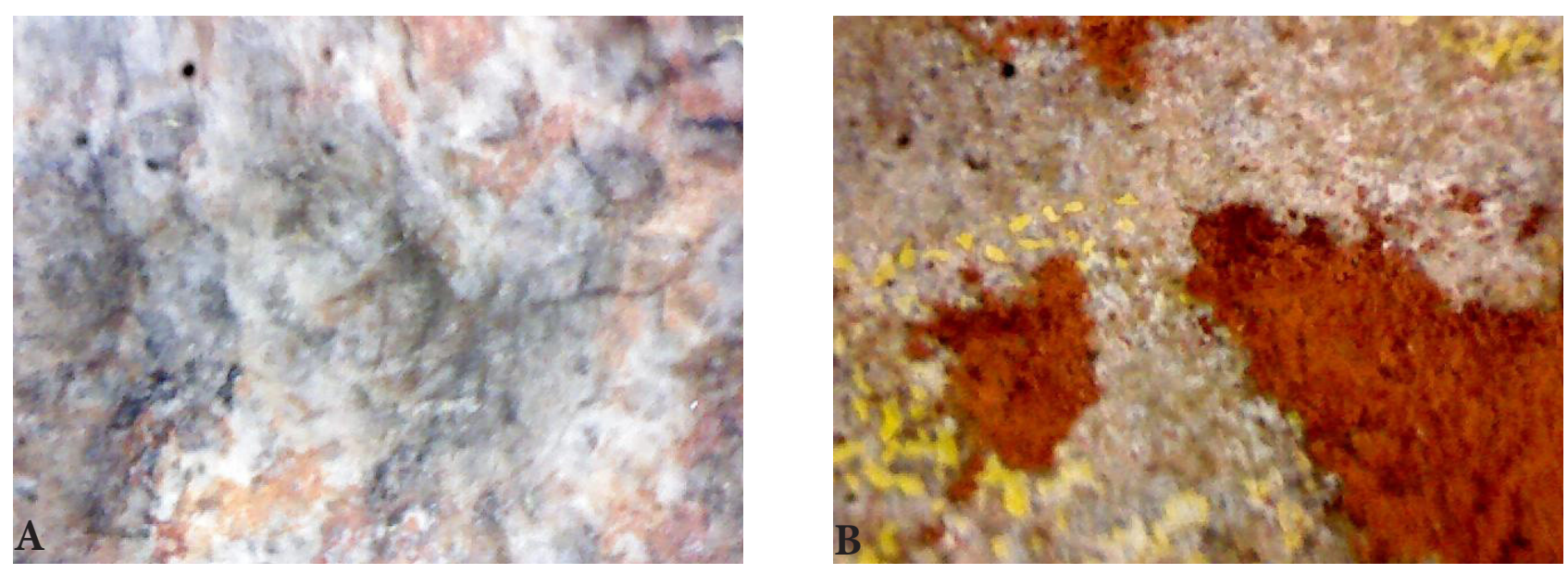

Figura 8: Fuligem recobrindo ocre vermelho (A); Pichação vermelha recobrindo pichação amarela (B) 


\section{CONSIDERAÇÕES FINAIS}

De nada adianta realizar trabalhos arqueométricos visando à documentação e elaboração de diagnósticos técnicos de conservação sem pensar em ações de conservação preventivas e de monitoramento de um sítio de arte rupestre de forma sistemática. Isto porque uma vez realizada a etapa de conservação, o sítio arqueológico necessita de visitas técnicas para verificar o avanço dos agentes degradantes (Lage et al., 2005). Uma das melhores formas de monitoramento é realizar o registro fotográfico da arte rupestre e do ambiente circundante, antes e após a etapa de intervenção, e repeti-la pelo menos duas vezes nos anos subsequentes à intervenção. A comparação entre as fotos de um mesmo painel tirada em períodos diferentes permite verificar a evolução do estado de conservação do sítio e propor ações preventivas. O monitoramento pode ser realizado também de forma presencial, observando os aspectos climáticos do sítio ao longo do ano, através de medidas como índice de pluviosidade, velocidade e direção dos ventos, temperatura da rocha e do ambiente, incidência da luz solar, entre outras. Como forma de bem documentar cada etapa de coleta desses dados, é importante o preenchimento de uma ficha técnica de conservação, a qual servirá para guiar o conservador em futuras intervenções. A ficha de conservação deve ser simples, mas conter todos os dados essenciais para acompanhamento dos sítios. As fichas elaboradas e utilizadas nos trabalhos de conservação, desenvolvidos pelo grupo de Arqueometria da UFPI, também são apresentadas aos condutores/guias de turistas que trabalham nos parques nacionais do Estado, principalmente o da Serra da Capivara e o de Sete Cidades. O papel desses especialistas é fundamental para os trabalhos de conservação, pois nós, pesquisadores, vamos aos sítios coletar nossos dados e depois voltamos para nossas atividades nas academias e quem fica no campo, em contato direto com os sítios arqueológicos, são eles e os moradores atuais dos seus entornos, portanto, é imprescindível integrá-los em nossas investigações, pois com certeza haverá uma maior riqueza de informações sobre os sítios e os fatos ocorridos neles.

Preocupação com a inclusão da comunidade atual nos trabalhos de conservação sempre aconteceu com nosso grupo de Arqueometria da UFPI, a exemplo citamos os primeiros trabalhos de conservação realizados na Serra da Capivara, quando formamos uma equipe de jovens da região para atuarem como auxiliares técnicos em conservação, os quais foram contratados pela FUMDHAM e auxiliaram nesta atividade por mais de uma década. Atualmente, apenas uma equipe reduzidíssima continua com estas atividades naquele parque. Nos demais sítios trabalhados também sempre foi feito um contato prévio com os moradores do entorno, a fim de apresentar a equipe, o projeto, e convidá-los para atuarem como auxiliares nas atividades da investigação. 


\section{REFERÊNCIAS}

ALENCAR, Wemerson J.; SANTOS, Francisco Eroni P.; CISNEROS, Juan Carlos; SILVA, João Henrique; FREIRE, Paulo Tarso C.; VIANA, Bartolomel C.; Spectroscopic analysis and X-ray diffraction of trunk fossils from the Parnaíba Basin, Northeast Brazil, Spectrochimica . Acta Part A, v. 135, p. 1052-1058, 2015.

ALVES, Marcos Jadiel. Análises Químico-mineralógicas de Pigmentos Pré-históricos de Sítios de São Miguel do Tapuio. (Dissertação) Mestrado em Química, Universidade Federal do Piauí, Teresina, 2014.

ALVES, Tetissuelma Leal. Análise química de pigmentos pré-históricos de sítios de Pedro II - PI. (Dissertação) Mestrado em Química, Universidade Federal do Piauí, Teresina, 2010.

ALVES, Tetissuelma L.; BRITO, Maria Auxiliadora M. L.; LAGE, Maria Conceição Soares Meneses; CAVALCANTE, Luis Carlos Duarte; FABRIS, José Domingos. Pigmentos de pinturas rupestres pré-históricas do sítio Letreiro do Quinto, Pedro II, Piauí, Brasil. Química Nova, v. 34, p. 181-185, 2011.

APPOLONI, Carlos Roberto; PARREIRA, Paulo Sérgio. Doze anos de atividades em arte e arqueometria no Laboratorio de Física Nuclear Aplicada da Universidade Estadual de Londrina. Revista Brasileira de Arqueometria, Restauração e Conservação, v. 1, n. 6, p. 301-304, 2007.

BROGGI, Alessandra; PETRUCCI, Elisabetta; BRACCIALE, Maria Paola; SANTARELLI, Maria Laura FT-Raman spectroscopy for quantitative analysis of salt efflorescences (pages 1560-1566), Journal of Raman Spectroscopy, v. 43, n. 11, p. 1560-1566, 2012.

BRUNET, Jacques ; VIDAL, Pierre ; VOUVE, Jean. Conservation de l'art rupestre: deux études, glossaire illustré, UNESCO, 1985, Paris.

BRUNET, Jacques; LAGE, Maria Conceiçào Soares Meneses; Premiers resultats de I'étudedespeintures rupestres de la Toca do Boqueirão do Sítio da Pedra Furada (Piauí-Brésil).9th Triennial Meeting: ICOMOS LRMH Comité del I-ICOM pour la conservation, $v$. 26-30, p. 782-792, 1990.

CAVAlCANTE, Luis Carlos Duarte. Arqueoquímica aplicada ao estudo de pigmentos, depósitos de alteração e paleossedimentos do Piauí. (Dissertação) Mestrado em Química, Universidade Federal do Piauí, Teresina, 2008.

CAVALCANTE, Luis Carlos Duarte; FARIAS FILHO, Benedito Batista; SANTOS, Livia Martins; FONTES, Laiane De Moura; LAGE, Maria Conceição Soares Meneses; FABRIS, José Domingos. Letreiro dos Tanques I e II: problemas de conservação e análises químicas de pinturas rupestres e eflorescência salina. ArqueologiaIberoamericana, v. 18, p. 3-13, 2013.

CAVAlCANTE, Luis Carlos Duarte; FERRARO, Lorena; HUGON, Paulette; LAGE, Maria Conceição Soares Meneses; FABRIS, José Domignos. Iron-bearing minerals of a rupestrian painting from the Manantial Solís site, Cardiel Lake, Patagonia, Argentina. Hyperfine Interactions, v. 238, p. 238-258, 2017.

FARIA, Dalva Lúcia A.; EDWARDS, Howell G. M.; AFONSO, Marisa Coutinho. Espectroscopia Raman: uma nova luz no estudo de bens culturais. Revista do Museu de Arqueologia e Etnologia, v. 12, p. 249, 2003.

FARIA, Dalva Lúcia A.; LOPES, Francisco N.; SOUZA, Luis Antonio C.; CASTELLO BRANCO, Helena David De Oliveira. Análise de pinturas rupestres do Abrigo do Janelão (Minas Gerais) por microscopia raman. Química Nova. v. 34, p. $1358-1364,2011$. 
FARIAS FILHO, Benedito Batista; LAGE, Maria Conceição Soares Meneses; LIMA, Rassius Alexandre Medeiros. Estudo químico de eflorescências salinas do sítio arqueológico Toca Exú do Jurubeba do Parque Nacional Serra da Capivara, Piauí, Brasil. Química Nova, v. 40, p. 1-6, 2017.

FERRAZ, Heloisa Bitú. O Sítio Rupestre de Santa Fé - CE: Documentação e Diagnóstico Técnico de Conservação. (Dissertação), Mestrado em Arqueologia, Universidade Federal do Piauí, Teresina, 2018

FONTES, Laiane de Moura. Caracterização espectroscópica de ocres, pinturas rupestres e depósitos salinos de sítios pernambucanos. (Dissertação) Mestrado em Química, Universidade Federal do Piauí, Teresina, 2010.

GUIDON, Niede; LAGE, Maria Conceição S. M. Sauvegarde des peintures préhistoriques d'un site de la Tradition Nordeste. INORA-França, n. 33, 2002.

HENNETIER, Luc; ALMEIDA, J. V.; CORREIA, Antonio M. S.; FERREIRA, Victor M. Efflorescence and its quantification in ceramic building materials, British Ceramic Transactions, v. 100, n. 2, p. 72-76, 2001.

IBIAPINA, Michel S. A Toca do Joel: um exemplo de aplicação da química no estudo e na preservação de um sítio arqueológico. (Dissertação) Mestrado em Química, Universidade Federal do Piauí, Teresina, 2007.

LAGE, Maria Conceição S. M. A conservação de sítios de arte rupestre. Revista do Patrimônio Histórico e Artístico Nacional, v. 33, p. 95-107, 2007.

LAGE, Maria Conceição. S. M. Abordagem dos Problemas de Conservação. Cadernos de Pesquisa, v. 1, n. 10, p. 31-70, 1992.

LAGE, Maria Conceição. S. M. Análise química de pigmentos de arte rupestre do Sudoeste do Piauí. Revista do Museu de arqueologia e Etnologia da USP, v. 2, p. 89-101, 1997

LAGE, Maria Conceição S. M. Étude Archéométrique de l>art rupestre du sud-est du Piauí - Brésil. (Tese), Université Paris 1, Paris, 1990.

LAGE, Maria Conceição S. M. A conservação de sítios de arte rupestre. Revista do Patrimônio Histórico e Artístico Nacional, v. 33, p. 95-107, 2007.

LAGE, Maria Conceição S. M.; BORGES, Jóina F.; ROCHA JÚNIOR, Simplício S. Sítios de Registros Rupestres: Monitoramento e Conservação. Mneme (Caicó. Online), Recife, v. 1, n.1, p. 01-24, 2005.

LAGE, Maria Conceição S. M.; SILVA, J. C.; MAGALHÃES, Sonia Maria C.; CAVALCANTE, Luis Carlos D.; SANTOS, Livia M.; FERRARO, Lorena. A restauração do Sítio Arqueológico Pedra do Castelo. CLIO. Série Arqueológica (UFPE), v. 24, p. 67-82, 2009.

LAGE, Maria Conceição S. M.; CAVALCANTE, Luis Carlos D.; KLINGELHÖFER, Goestar; FABRIS, José Domigos: Insitu $57 \mathrm{Fe}$ Mössbauer characterization of iron oxides in pigments of a rupestrian painting from the Serra da Capivara National Park, in Brazil, with the backscattering Mössbauer spectrometer MIMOS II. Hyperfine Interact. V. 237, p. 49, 2016.

MAGUREGUI, Maite; KNUUTINEN, Ulla; MART'INEZ-ARKARAZO, Irantzu; CASTRO, Kepa; MADARIAGA, Juan M.; Thermodynamic and spectroscopic speciation to explain the blackening process of hematite formed by atmospheric SO2 impact: The case of Marcus Lucretius House (Pompeii), Analytical Chemistry, v. 83, n. 9, p. 3319-3326, 2011.

PÉREZ-AlONSO, Maite; CASTRO, Kepa; ÁlVAREZ, Maria; MADARIAGA, Juan Manuel. Scientific analysis versus restorer's expertise for diagnosis prior to a restoration process: the case of Santa Maria Church (Hermo, Asturias, North of Spain), Analytical Chimica Acta, v. 524, p. 379-389, 2004. 
QUEIRÓS, Agnelo F. Os grafismos rupestres do Médio-Baixo Jaguaribe, Ceará: documentação, análise e contextualização preliminar. (Dissertação) Mestrado em Química, Universidade Federal do Piauí, Teresina, 2016.

RODRIGUES, Wallonilson Veras. Análise de pinturas rupestres por espectroscopia Raman em sítios arqueológicos do Parque Nacional de Sete Cidades. (Dissertação) Mestrado em Química, Universidade Federal do Piauí, Teresina, 2014.

ROLDÁN, Clodoaldo; MURCIA-MASCARÓS, Sonia; FERRERO, José; VILLAVERDE, Valentin; LÓPEZ, Esther; DOMINGO, Inês.; MARTINEZ, Rafael; GUILLEM, Pere Miquel. Application of Field portable EDXRF spectrometry to analysis of pigments of Levantine rock art. X ray Spectrometry, v. 39, n. 3, p. 243-250, 2010.

SAIKIA, Bhaskar J.; PARTHASARATHY, Gopalaswami; SARMAH, N. C.; Fourier transform infrared spectroscopic estimation of crystallinity in $\mathrm{SiO}_{2}$ based rocks, Bulletin of Materials Science, v. 31, p. 775-779, 2008.

SANTOS, Livia Martins Análise Químico-mineralógica de pinturas rupestres do sítio Salão dos Índios - Piauí - Brasil. (Dissertação) Mestrado em Química, Universidade Federal do Piauí, Teresina, 2010.

SANTOS, Livia Martins; OLIVEIRA, F. M.; FARIAS FILHO, Benedito Batista; FONTES, Laiane de Moura; LAGE, Maria Conceição S. M.; SILVA, Heralda Kellis S. B.; CAVALCANTE, Luis Carlos Duarte; FABRIS, José Domigos.Chemical and mineralogical characteristics of the pigments of archaeological rupestrian paintings from the Salão dos Índios site, in Piauí, Brazil. Journal of Archaeological Science: Reports, p. 792-797, 2018.

SOUSA, Emanuel Martins. Parque Nacional de Sete Cidades - Piauí - Brasil: exame e análise química de pigmentos préhistóricos. (Dissertação) Mestrado em Química, Universidade Federal do Piauí, Teresina, 2002.

SOUZA, Maria Cleidiane P. Estudo de Pigmentos Pré-históricos dos Sítios: Pedra Furada dos Picos I e Letreiro do Ninho do Urubu. (Dissertação) Mestrado em Química, Universidade Federal do Piauí, Teresina, 2009.

VANDENABEELE, Peter; DONAIS, Mary Kate. Mobile Spectroscopic Instrumentation in Archaeometry Research, Applied Spectroscopy, v. 70, p. 27-41, 2016. 